\title{
Responsible conduct of research: enhancing local opportunities.
}

\author{
Erisa S Mwaka
}

Anatomy Department, School of Biomedical Sciences, College of Health Sciences, Makerere University. P.O. Box 7072, Kampala, Uganda.

\begin{abstract}
Introduction: Research integrity is the foundation of credible research and a pre-requisite for a successful academic research environment. Lately, a lot of revelations of fraud and other unacceptable behaviour in research have been highly publicized in scientific journals and mass media. Whereas institutions in developed countries have developed guidelines and regulations to ensure responsible conduct of research and appropriately deal with cases of research misconduct, low- and middle-income countries seem to be lagging behind. In Uganda, there seems to be lack of coordinated efforts to address the problem of research misconduct both at the national and institutional level.

Objective: To propose a framework for fostering scientific integrity and deterring misconduct in research in Ugandan research and academic institutions.

Methods: A review of literature on scientific integrity, scientific misconduct, responsible conduct of research, and international ethical guidelines was done.

Results: Basing on the 2012 Inter-Academy Council policy report, initiatives to promote responsible conduct of research in Ugandan research and academic institutions are proposed.

Conclusion: With the proposed framework, an honest and trustworthy research enterprise in Uganda based on principles of scientific integrity is envisioned.

Keywords: Research misconduct, scientific integrity, responsible conduct of research.

DOI: https://dx.doi.org/10.4314/ahs.v17i2.36

Cite as: Mwaka ES. Responsible conduct of research: enhancing local opportunities. Afri Health Sci. 2017;17(2): 584-590. https://dx.doi. org/10.4314/abs.v17i2.36
\end{abstract}

\section{Introduction}

Research integrity is the foundation of credible research and a pre-requisite for a successful academic research environment. Research integrity requires adherence to professional standards prescribed by oversight bodies such as governmental agencies, funding agencies and professional associations as well as adherence to ethical principles and values that promote responsible conduct of research (RCR) ${ }^{1}$. With globalization of research, a lot of foreign funded collaborative studies are increasingly being conducted in developing countries. Therefore, standardized
Corresponding author:
Erisa S Mwaka
Anatomy Department,
School of Biomedical Sciences,
College of Health Sciences,
Makerere University
P.O. Box 7072, Kampala, Uganda.
Tel: +256752575050
Email: erisamwaka@yahoo.com

approaches to interpretation and implementation of the principles and values that underlie RCR need to be clearly defined and promoted ${ }^{1}$.

Despite developing and adoption of documents and guidelines that promote RCR by research and academic institutions there seems to be no downward trend in the incidence of scientific misconduct. On the contrary studies show that misconduct is on the increase ${ }^{2}$. Lately, a lot of revelations of fraud and other unacceptable behaviour in research have been highly publicized in scientific journals and mass media ${ }^{3-5}$. These revelations not only damage the credibility of individual perpetrators, they also have negative consequences on the integrity of the institution in which the research was conducted.

Irresponsible behaviour can negatively impact research in four ways. It can: challenge the reliability of the research record, weaken trust between professional colleagues and public trust in researchers, waste research funds, and culminate in decisions that cause public and/or personal harm ${ }^{6}$. Scientific misconduct can also have far-reaching implications on policy and clinical practice as is evidenced 
from a case published by the British Medical Journal in $2013^{5}$.

Whereas institutions in developed countries have developed guidelines and regulations to ensure RCR and appropriately deal with cases of research misconduct, lowand middle-income countries seem to be lagging behind. In Africa, even countries like South Africa ${ }^{1}$ and Nigeria ${ }^{7}$ which have had fairly well established research ethics review environments for decades lack structures for the prevention, detection, investigation and correction of research misconduct. In addition, very little empirical research on scientific integrity has been done in Africa ${ }^{7}$. Therefore it is difficult to deny or accept that research in low- and middle-income countries is conducted in concordance to international ethical standards or that scientific misconduct is rare.

In Uganda, there seems to be lack of coordinated efforts to address the problem of research misconduct both at the national and institutional level. This topic has also not featured prominently in public debate. The Uganda National Council for Science and Technology (UNCST) was established in 1990 by the Uganda National Council for Science and Technology $\mathrm{act}^{8}$ and among other functions it is mandated to oversee and coordinate research and development in Uganda'. Technically, UNCST is responsible for formulating ethical guidelines, accrediting research ethics committees (REC) and ensuring the ethical conduct of research, but it is silent on research integrity. Although there are UNCST guidelines for health research ethics $^{9}$, national guidelines for the promotion of RCR are lacking. There is no oversight body or association of interested parties mandated to prevent, directly address, investigate and correct allegations or cases of scientific misconduct even at the institutional level. However some professional bodies like the Uganda Medical and Dental Practitioners' Council, the Pharmacy council, the Allied health professional council and the Nurses and Midwives Council regulate and enforce adherence to professional standards. They are mandated to investigate allegations of professional misconduct and prescribe appropriate sanctions where necessary. There is need to establish both national and institutional offices of research integrity to handle allegations of research misconduct in the country.

For the meantime as the debate goes on whether to establish national and institutional offices of research integrity in Uganda or not, centres of higher learning and research institutions should adopt the 'Singapore statement' ${ }^{\text {'10 }}$ as a reference point for a shared understanding of research integrity in the country. The Singapore statement emphasizes the principles and professional responsibilities that define research integrity irrespective of national and cultural disparities in standards for scientific research. ${ }^{10}$ The statement enunciates four basic principles: honesty in all aspects of research; accountability in the conduct of research; professional courtesy and fairness in working with others; and stewardship of research on behalf of others $^{10}$. It also enumerates 14 fundamental responsibilities for researchers and research institutions. In this paper, we propose a framework for promoting scientific integrity and the responsible conduct of research in Uganda.

\section{Methods}

A review of relevant literature on strategies for enhancing RCR was done. A internet search was made for articles on RCR; documented cases of gross research misconduct; selected international guidelines and policy documents on research integrity; and the UNCST guidelines for conducting research involving human participants.

\section{Results and discussion}

A framework for fostering integrity and deterring scientific misconduct in research in Ugandan research and academic institutions is proposed basing on the 2012 Inter-Academy Council policy report ${ }^{11}$.

"Science is founded on values of integrity and trust" 12 . Therefore institutions are obligated to take responsibility for promoting scientific integrity and RCR. Research misconduct should be approached from the perspective of professional standards and not professional ideals. Efforts to improve integrity in research need to begin with the careful study of digressions from professional standards, their causes, and measures that might reasonably be expected to change behaviour ${ }^{6}$. Therefore initiatives to promote RCR should be multipronged and require contributions from the researcher, academic and/or research institutions, national/international regulatory bodies and funding agencies as discussed below.

\section{Individual researchers}

Researchers are motivated to maintain high standards because results from their scientific work not only brings them individual fame, honour and international recognition, it also improves on the institution's standing and attracts more research funding opportunities. 
Researchers have the fundamental responsibility for upholding standards of responsible conduct in research. They should employ the expected standards of their fields, observe applicable legislation and regulations, be willing to share results with others, and be agreeable on the standards to be observed in multidisciplinary collaborations. Researchers have an obligation to themselves, their colleagues, and society to avoid both the egregious misbehaviours of falsification, fabrication, and plagiarism and other questionable research practices that can undermine the research enterprise. Researchers have a responsibility to uphold high standards of responsible conduct and to act appropriately when they witness or suspect irresponsible conduct. Therefore it is imperative that research is conducted ethically and with great integrity.

In international collaborative research, partners need to agree on RCR guidelines and procedures to address research misconduct in the initial stages of the research. Any suspected deviation from set standards, in particular research misconduct should be immediately brought to the attention of the project leader or concerned administrator, in order for it to be investigated according to the policies and procedures of the partner with the primary responsibility, while respecting the laws and sovereignity of the States of all participating parties ${ }^{13}$.

Peer reviewers need to assess other people's work professionally, fairly and promptly. Peer review seeks to ensure the relevance of the communication, that conclusions are supported by evidence, and that the findings are of value. Researchers should keep clear, accurate and secure records of their research data and primary material for easy verification and replication by others. Where necessary, they should share data with others, but they should first establish priority or ownership claims before disseminating their findings.

\section{Funding agencies}

Local and foreign sponsors of research should provide support to researchers and research institutions to ensure that they conduct research responsibly, without compromising quality or integrity. Funding agencies should support research and academic institutions to promote research integrity through education and training programs on RCR. They should also encourage these institutions to develop mechanisms and procedures to respond to research misconduct. Since funding bodies have no juris- diction on the quality and integrity of research they have not funded, it is imperative that the standards set are universally applicable and in line with national and international norms and guidelines.

\section{Registration of clinical trials and other major re-} search studies: Clinical trials should be registered so that their progress can be tracked and their outcomes published ${ }^{14}$. Under-reporting research is scientific misconduct and has dire consequences ${ }^{15}$. It skews the utility of drugs and devices by overestimating the benefits of treatment and underestimating their undesirable effects. This distorts the evidence base for clinical decisions thus putting patients at risk and wasting valuable health care resources ${ }^{14}$. In some countries, there are powerful campaigns calling on pharmaceutical companies to publish all the information they have about licensed drugs ${ }^{16}$. This registration should also be extended to include major publicly or privately funded research projects.

\section{Institutional strategies}

Academic and scientific community sensitization: There is also need to sensitize the academic and scientific community about scientific misconduct ${ }^{7}$. Research institutions and professional societies, working with government, should make efforts to ensure that professional standards for RCR are clear, accessible, taught, and monitored. Examples of such efforts include research ethics review regulations, misconduct policies, publication rules and standards for reporting clinical trials.

Research mentors, senior faculty and unit heads are responsible for defining, explaining, exemplifying, and ensuring adherence to the value systems of their institutions. Administrative officials of research institutions should put in place a balanced reward system that appropriately recognizes research quality, integrity, teaching and mentorship ${ }^{17}$.

\section{Enhanced monitoring of research and scholar-} ly works: One of the major outputs of research is the publication of findings in peer reviewed journals. Thus, the use of retracted scientific articles by peer reviewed journals can be a measure of the research misconduct. A comprehensive survey by Grieneisen and Zhang showed that since 2001 the number of publication retracted from scholarly literature has been steadily increasing, with $20 \%$ being due to alleged research misconduct and $42 \%$ due to 
questionable research practice ${ }^{18}$. Studies further indicate that retraction rates are higher in high impact journals. This situation poses serious questions about the trustworthiness and integrity of published data. To curb this exponential rise in scientific misconduct, it is necessary that editors, researchers and research institutions become more vigilant and institute concerted efforts to address this problem right through the entire research process. In the absence of an office of research integrity in Ugan$\mathrm{da}$, enhanced monitoring of research and scholarly works can be achieved by empowering existing research ethics committees (REC) through better funding and personnel training to ensure that they perform their oversight function more efficiently. Of most importance is the active and passive monitoring of approved research studies to ensure compliance with regulatory standards ${ }^{7}$.

Enhanced monitoring and audit: Academic and research institutions, principal investigators and other research group heads should devise means of supervising and monitoring the research process. This can be in form of random spot audits of projects and programs, use of electronic database systems, dynamic forms and protocol tracking software, use of digital technology to check for plagiarism, digital image analysis to detect inappropriate manipulation of images, and forensic examination to determine the validity of datasets ${ }^{19,20}$. Academic institutions should invest in plagiarism-detection software to discourage and detect plagiarism. This software is now routinely used by academic institutions and journals. It should be made mandatory for all students to first submit their scholarly work to such programs before handing them to their supervisors. This will encourage students to desist from irresponsible authorship practices. Evidence shows that this is already having a positive impact on the fight against plagiarism ${ }^{21}$.

Education, training and mentorship: Since it is difficult and expensive to detect, report and investigate scientific misconduct, it is prudent that misconduct is prevented in advance rather than regulating it afterwards ${ }^{22}$. This can be best achieved through education and training. The primary goal of RCR education is to promote and improve ethical conduct of research and decrease scientific misconduct. RCR education should foster a research culture where free discussion about RCR is expected and acceptable. The focus should be to promote among research- ers' discussions, active engagement and critical thinking about the ethical conduct of research, and foster positive attitudes towards promoting $\mathrm{RCR}^{23}$. Health research ethics and responsible conduct of research should be incorporated into training curriculums at both undergraduate and graduate levels. Scientists should receive regular refresher courses and continuing professional education on ethics, research integrity and the responsible conduct of research ${ }^{7}$. This should be mandatory and can be delivered through locally organized training or through web based training from reputable sites like National Institutes of Health office of extramural research ${ }^{24}$ and, Training and Resources in Research Ethics Evaluation (TRREE) ${ }^{25}$. In this regard, Makerere University College of Health Sciences (MakCHS) was awarded a grant to develop a Master of Science degree in International Health Research Ethics (IHRET). Among other activities, the IHRET program is conducting trainings and short courses on RCR, research ethics and clinical ethics. All stakeholders in the research enterprise should be encouraged to undertake these short courses with a long term goal of producing researchers who are well grounded in research ethics.

Another strategy is the mentorship of new researchers and students in order to produce good and successful researchers. On the other hand, mentors too require some training if they are to convey adequately and continuously to the mentees ${ }^{12}$. In a study of 2000 laboratory directors by the US Office for Research Integrity (ORI), only 33\% reported having been well prepared by their mentors to become good mentors ${ }^{26}$. To illustrate the importance of mentorship, an example is given of another study conducted by the US ORI on the role played by mentors in cases of trainee research misconduct. It was found that $73 \%$ of mentors/supervisors had not looked at the primary data generated by their trainees; $62 \%$ had little appreciation of the conduct of research they were presumably supervising and had not set adequate supervisory standards for their mentees, such as maintaining laboratory notebooks; and mentors seemed to pay little attention to mentees' reports because of high stress levels ${ }^{27}$. Therefore institutional leaders need to play a more active role with researchers to build institutional programs that teach mentors how to mentor. In order to reduce the incidence of trainee misconduct or reduce its impact, mentors must ensure that they regularly review trainee raw/primary data as a preventive strategy of reducing opportunities 
for trainee misconduct; set appropriate standards, rules and procedures for collecting, recording and maintaining data, and ensure that they are implemented and enforced; and pay more attention to potential stressors that might significantly affect the performance of their trainees ${ }^{27}$.

\section{Developing an appropriate institutional culture of} integrity in research: Academic and research institutions can ensure research integrity by creating an environment that promotes RCR through adopting standards of excellence, trustworthiness and lawfulness ${ }^{28}$. This can be achieved by creating an organizational culture that encourages and insists on scientific integrity and $\mathrm{RCR}^{12}$. In view of the challenges posed by the factors that supposedly culminate in scientific misconduct, universities and research institutions have to devise and adopt creative strategies to curb this vice. University leaders and senior faculty need to devote time to speak, and/or attend meetings or seminars that address scientific and academic integrity. Secondly, bureaucracy should be minimized, and researchers must be availed with adequate support systems and infrastructure to mitigate situations that may promote irresponsible research practices ${ }^{12}$. Third, active fostering of academic collegiality and communication with in the institution can contribute to a conducive environment for grooming 'good' and 'successful' responsible researchers ${ }^{12}$.

Adopting and developing research codes and policies at institutional level: There are many internationally and nationally recognized codes of conduct that promote RCR, for example, the Singapore statement, Helsinki Declaration and the UNCST national guidelines for research involving humans as research participants $^{29-31}$, however they are not always extensively read or understood $^{19}$. Researchers must be aware of these codes of research conduct and institutions have a responsibility of promoting that awareness. Studies show that scientists who read their institutional policies are more like to report cases of misconduct ${ }^{32}$.

\section{Enhanced measures for detection of misconduct:} Universities should focus on a limited number of high quality publications to reduce the quantitative drive that pushes scientists into behaving irresponsibly in a bid to enhance their individual publication record ${ }^{19}$.

Despite there being several international, national and in- stitutional codes, guidelines, laws, rules and regulations that ensure that research is conducted with the highest integrity the incidence of scientific misconduct is on the rise $^{2}$. This might be an indication that human character and circumstance have a bearing on how people conduct themselves in research ${ }^{33}$.

Institutions should establish effective mechanisms for dealing with allegations of research misconduct promptly, impartially and confidentially ${ }^{11}$. This can be achieved through the establishment of national and institutional oversight bodies responsible for developing national guidelines on research integrity as well as preventing, identifying and investigating cases of scientific misconduct $^{7}$. In this regard, MakCHS is in the process of developing a policy on scientific integrity. Allegations of research misconduct should be thoroughly investigated. Those found guilty ought to be punished and those vindicated should be protected. To avert gross violations of research integrity, the scientific community must be alert and more vigilant at preventing, detecting and admonishing scientific misconduct ${ }^{7}$. Institutions must devise mechanisms of identifying circumstances or situations that could culminate in incidents or allegations of research misconduct early. Institutions should appreciate and acknowledge the contribution of whistle-blowers in the maintenance of scientific integrity. Though there is legislation prohibiting retaliation ${ }^{34}$ against whistle-blowers in some countries, a majority of whistle-blowers suffer retaliatory attacks for their actions ${ }^{35}$. This causes many would-be whistle-blowers to refrain from the act. Institutions should put in place protective measures against retribution. An institutional office should be established to receive these allegations and try to solve them before official inquiries are made ${ }^{33}$. Exposing misconduct plays a significant role in maintaining the integrity of scientific research. Institutional policies and regulations to address allegations of scientific fraud must include provisions for the protection of the accuser and the accused (in case they are exonerated $)^{36}$.

\section{Conclusion}

Researchers and research institutions often have an assumption that scientific research can effectively regulate its own behavior. They assume that misconduct cannot be as rampant since it is kept in check by peer review and self-regulation due to the competitive nature of the scien- 
tific community ${ }^{6}$. Revelations of research misconduct are embarrassing for academic institutions, journals and have far-reaching consequences on the research enterprise in general and the implicated individual researcher ${ }^{1}$. Most, if not all academic and research institutions in Uganda have no designated offices for research integrity. There are no standard guidelines for promoting research integrity; institutions have the autonomy to develop their own ad hoc mechanisms of responding to allegations of scientific misconduct. However there is the reluctance of individual institutions to effectively handle cases of scientific misconduct especially if it concerns high profile researchers. It is well documented that institutions tend to be complacent when it comes to investigating unethical behaviour particularly when the ramifications are unfavourable to the institution; they tend to have a conflict of interest $\mathrm{t}^{37}$.

Ideally, when allegations of scientific misconduct arise, there should be institutional mechanisms of dealing with the allegations. Should the internal remedies be exhausted without resolution then an external entity can be sought for fair and equitable adjudication. For external adjudication there is need for a centralised/national body or office of research integrity that is mandated with establishing national guidelines on RCR and appropriate procedures for responding to cases of misconduct. The responsibilities of the national body should include developing explicit policies, procedures and regulations for detection and response to scientific misconduct and the RCR; supervision, monitoring and providing technical assistance to institutional offices that respond to allegations of research misconduct; and implementing educational and training activities to promote RCR, research integrity, prevent scientific misconduct, and improve the handling of allegations of scientific misconduct ${ }^{38}$.

We propose multiple disciplinary involvements in establishing a Ugandan framework for RCR, including research and academic institutions, professional organizations, government departments, funders/sponsors of research activities and research consortia. Such a framework could then be maintained and implemented by a centralized national body. As a prelude to this, empirical research on scientific integrity should be conducted to document the presence or absence, and magnitude of scientific misconduct in the country. Academic and research institutions ought to encourage open debate on this topic as a way of raising awareness in the wider scientific community, and possibly arouse public interest. We envision an honest and trustworthy research enterprise in Uganda based on principles of scientific integrity ${ }^{11,13}$.

\section{Competing interests}

The author declares no conflict of interest.

\section{References}

1. Rossouw TM, Van Zyl C, Pope A. Responsible conduct of research: Global trends, local opportunities. South African Journal of Science. 2014;110(1-2):1-6.

2. Resnik DB, Dinse GE. Scientific retractions and corrections related to misconduct findings. Journal of medical ethics. 2012:medethics-2012-100766.

3. Marc Hauser engaged in research misconduct. Harvard Magazine [serial on the Internet] [Available from: http:/ / harvardmagazine.com/2012/09/hauser-research-misconduct-reported.

4. Pelley S. Deception at Duke: Fraud in cancer care? [Available from: http://www.cbsnews.com/830118560_162-57376073/deception-at-duke-fraud-in-cancer-care/.

5. Wise J Boldt: the great pretender. BMJ: British Medical Journal. 2013;346.

6. Steneck NH. Fostering integrity in research: Definitions, current knowledge, and future directions. Science and engineering ethics. 2006;12(1):53-74.

7. Okonta P, Rossouw T. Prevalence of scientific misconduct among a group of researchers in Nigeria. Developing world bioethics. 2013;13(3):149-57.

8. Uganda National Council for Science and Technology Act. 1990. Cap. 209 of the laws of Uganda.

9. UNCST. Uganda National Council for Science and Technology: National Guidelines for Research Involving Humans as Research Partcipants. Kampala, Uganda2014. 10. Resnik DB, Shamoo AE. The singapore statement on research integrity. Accountability in research. 2011;18(2):715 .

11. Inter-Academy Council/IAP. Responsible conduct in the global research enterprise: A policy report. 2012 Amsterdam: Bejo Druk \& Print, Alkmaar; 2012: Inter Academy Council; 2012 [Available from: http://www.interacademycouncil.net/24026/GlobalReport.aspx.

12. Horn L. Promoting responsible research conduct in a developing world academic context. South African Journal of Bioethics and Law. 2013;6(1):21-4.

13. European Science Foundation. Fostering research integrity in Europe: A report by the ESF Member Organi- 
sation Forum on research integrity 2011 [Available from: http://www.esf.org/fileadmin/Public_documents/Publications/Code_Conduct_ResearchIntegrity.pdf.

14. Chalmers I, Glasziou P, Godlee F. All trials must be registered and the results published. BMJ: British Medical Journal. 2013;346.

15. Chalmers I. Underreporting research is scientific misconduct. JAMA internal medicine. 2010;263:1405-8.

16. Hawkes N. Drug companies should release data from all trials of licensed drugs, say MPs. BMJ. 2013;346:f321.

17. Committee on Science Engineering and Public Policy (U.S.). Panel on Scientific Responsibility and the Conduct of Research. Responsible science: Ensuring the integrity of the research process. Washington, D.C.: National Academy Press; 1992.

18. Grieneisen ML, Zhang M. A comprehensive survey of retracted articles from the scholarly literature. PloS one. 2012;7(10): e44118. PubMed

19. Farthing MJ. Research misconduct: A grand global challenge for the 21st Century. Journal of gastroenterology and hepatology. 2014;29(3):422-7.

20. Hicks J. Opinion: Ethics Training in Science; The $\mathrm{NIH}$ has required researchers to receive instruction about responsible conduct for more than 20 years, but misconduct is still on the rise 2013 [Available from: http:/ /www. the-scientist.com/?articles.view/articleNo/35543/title/ Opinion--Ethics-Training-in-Science/.

21. Jump P. Turnitin is turning up fewer cases of plagiarism. Times Higher Education. 2013;4 Apr.

22. Fox MF, Braxton JM. Self-regulation and social control of scientific misconduct: Roles, patterns, and constraints. In: Braxton JM, editor. Perspectives on scholarly misconduct in the sciences. Columbus, $\mathrm{OH}$ : The Ohio State University Press; 1999. p. 315-30.

23. Kalichman M. Rescuing responsible conduct of research (RCR) education. Accountability in research. 2014;21(1):68-83.

24. National Institute of Health Office of Extramural Research. Protecting human research participants [Available from: https://phrp.nihtraining.com/users/login. php.

25. TRREE. Training and Resources in Research Ethics Evaluation (TRREE) [Available from: http://elearning. trree.org/.
26. Rodbard D. Survey of research integrity measures utilized in biomedical research laboratories, report prepared for ORI, 20032003 [Available from: http://ori. hhs.gov/images/ddblock/intergity_measures_final_report_11_07_03.pdf.

27. Wright DE, Titus SL, Cornelison JB. Mentoring and research misconduct: An analysis of research mentoring in closed ORI cases. Science and engineering ethics. 2008;14(3):323-36.

28. Council of Canadian Academies. The expert panel on research integrity. Honesty, accountability and trust: Fostering research integrity in Canada. Ottawa: Council of Canadian Academies; 2010.

29. Singapore Statement on Research Integrity Singapore: Second World Conference on Research Integrity; 2010 [Available from: http://www.singaporestatement.org/. 30. UNCST. Uganda National Council for Science and Technology: National Guidelines for Research Involving Humans as Research artcipants. 2007.

31. The World Medical Association. (2013). Declaration of Helsinki 2013 [Accessed 19/02/2014]; Available from: URL: http:// www.wma.net/e/ethicsunit/helsinki.htm., (2013).

32. Wells JA. Final report: Observing and reporting suspected misconduct in biomedical research. Rockville, MD: The Office of Research Integrity.; 2008.

33. Kornfeld DS. Perspective: Research misconduct: The search for a remedy. Academic Medicine. 2012;87(7):877-82. 34. Department of Health and Human Services. Public health service standards for the protection of research misconduct whistleblowers. 2012. Contract No.: $7 /$ July. 35. Research Triangle Institute. Consequences of whistleblowing for the whistleblower in misconduct in science cases. Washington, DC: Office of Research Integrity; 1995.

36. Lubalin JS, Matheson JL. The fallout: What happens to whistleblowers and those accused but exonerated of scientific misconduct? Science and engineering ethics. 1999;5(2):229-50.

37. Rhodes R, Strain JJ. Whistleblowing in academic medicine. Journal of medical ethics. 2004;30(1):35-9.

38. US Department of Health and Human Service: Office of Research Integrity. [Available from: http://ori. hhs.gov/. 